\title{
Investigation on Contact Resistance of Amorphous Indium Gallium Zinc Oxide Thin Film Transistors with Various Electrodes by Transmission Line Method
}

\author{
Sang Yeol Lee ${ }^{\dagger}$ \\ Department of Semiconductor Engineering, Cheongju University, Cheongju 360-764, Korea
}

Received April 28, 2015; Revised May 3, 2015; Accepted May 7, 2015

\begin{abstract}
Contact resistance of interface between the channel layers and various S/D electrodes was investigated by transmission line method. Different electrodes such as Ti/Au, a-IZO, and multilayer of a-IGZO/Ag/a-IGZO were compared in terms of contact resistance, using the transmission line model. The a-IGZO TFTs with a-IGZO/Ag/a-IGZO of S/D electrodes showed good performance and low contact resistance due to the homo-junction with channel layer.
\end{abstract}

Keywords: Amorphous oxide semiconductor, Source and drain electrode, a-IGZO, Transparent conductive oxide

\section{INTRODUCTION}

Amorphous oxide thin film transistors (TFTs) | have advantages of high mobility and low temperature in device fabrication. Since they also have high transparency in the visible range, they were regarded as transparent TFTs (TTFTs) [1-3]. Amorphous InGaZnO (a-IGZO) is regarded as channel layer of TTFT due their good performance, transparency, and stability [4]. There are many studies of channel layer as well as source and drain (S/ D) electrodes of TTFTs, because the S/D electrodes have an effect on the electrical performance and transparency of TTFTs. Transparent conductive oxides (TCOs) are suitable for the S/D electrodes of TTFTs due to their high optical bandgap (>3.5 eV), good electrical conductivity, and high optical transparency of $80 \%$ in visible-infrared. Particularly, amorphous indium zinc oxide (a-IZO) was used for S/D electrode of ZnO TTFTs, due to high transparency and lower resistivity. Although there have been many researches on S/D electrodes of ZnO based TFTs, regarding their transparency, resistivity, and contact problem with channel layer, the relationship between the stability of ZnO based TFTs

${ }^{\dagger}$ Author to whom all correspondence should be addressed: E-mail: sylee@cju.ac.kr

Copyright $@ 2015$ KIEEME. All rights reserved. This is an open-access article distributed under the terms of the Creative Commons Attribution Non-Commercial
License (http://creativecommons.org/licenses/by-nc/3.0) which permits unrestricted noncommercial use, distribution, and reproduction in any medium, provided the original work is properly cited. under the various stress and S/D electrodes is still unknown [5-8]. In this paper, the influence of S/D electrodes on contact resistance of a-IGZO TFTs has been investigated by transmission line method (TLM). The change in S/D electrode has an effect on interfacial traps and energy barrier between the channel layer and S/D electrodes. The different contact resistance in electrical performance of a-IGZO TFTs, depending on S/D electrode, has been studied using TLM.

\section{EXPERIMENTAL}

The a-IGZO TFTs were fabricated by using a thermally grown $\mathrm{SiO}_{2}$ thin film on highly p-type $\mathrm{Si}(001)$ substrate as both substrate and gate electrode. The a-IGZO layer $(50 \mathrm{~nm})$ was deposited using rf magnetron sputtering with the channel width (250 $\mu \mathrm{m})$ and channel length $\left(\mathrm{L}_{\mathrm{ch}}\right)$ ranging from $100 \mu \mathrm{m}$ to $650 \mu \mathrm{m}$, as shown in Fig. 1. All devices were annealed at $350^{\circ} \mathrm{C}$ for $1 \mathrm{~h}$ in N2 ambient. Then, the different electrode was deposited and patterned using lift-off method. The Ti $(10 \mathrm{~nm})$ and $\mathrm{Au}(60 \mathrm{~nm})$ as S/ D electrodes was deposited by e-beam and thermal evaporation, respectively. The a-IZO electrodes and a-IGZO layer of a-IGZO/ Ag/a-IGZO electrodes were grown by rf magnetron sputter method, and the Ag was deposited using the thermal evaporation. Figure 2 shows optical microscope image of real TLM patterned devices. Transfer characteristics are measured at a drain voltage 


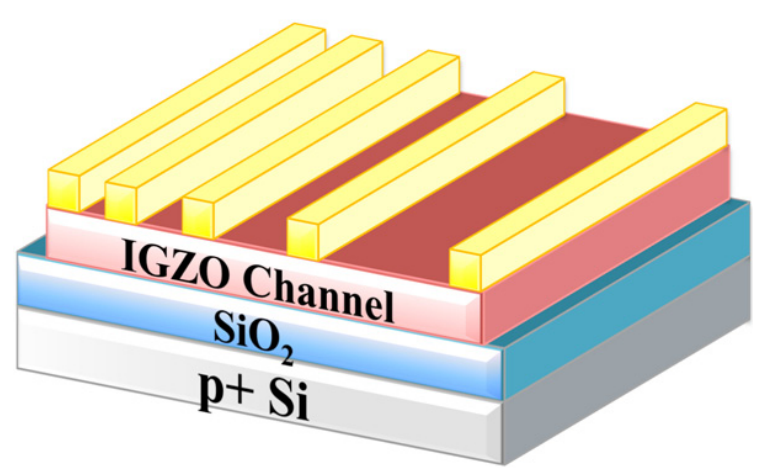

Fig. 1. Schematic diagram of a-IGZO TFT with TLM patterned electrodes.

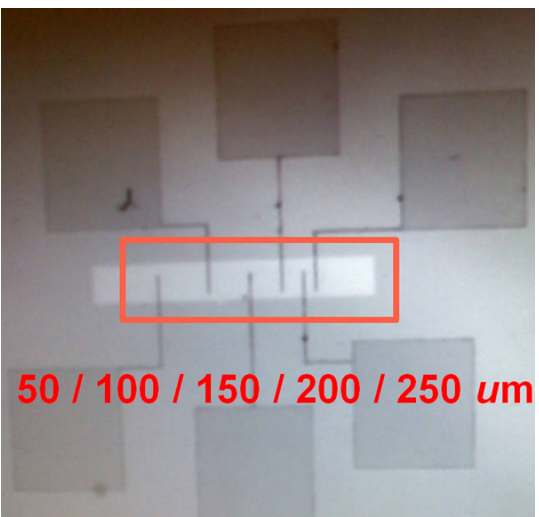

Fig. 2. Optical microscope image of a-IGZO TFT with TLM patterned electrodes.

VDS of $5.1 \mathrm{~V}$ with a single-sweep gate voltage $\mathrm{V}_{\mathrm{GS}}$ mode from -20 to $40 \mathrm{~V}$. The Vth is defined as the $\mathrm{V}_{\mathrm{GS}}$ value, where a drain current IDS of $5 \mathrm{nA}$ flows.

\section{RESULTS AND DISCUSSION}

Figure 3 shows the transfer curves of a-IGZO TFTs depending on $\mathrm{L}_{\mathrm{ch}}$ with (a) Ti /Au electrodes, (b) a-IZO electrodes, and (c) a$\mathrm{IGZO} / \mathrm{Ag} / \mathrm{a}-\mathrm{IGZO}$ electrodes. The inset shows output characteristics of a-IGZO TFTs measured at $\mathrm{V}_{\mathrm{GS}}=15 \mathrm{~V}$ with different $\mathrm{L}_{\mathrm{ch}}$. As $\mathrm{L}_{\mathrm{ch}}$ increases, the off current $\left(\mathrm{I}_{\text {off }}\right.$ ) and subthreshold swing (SS) were increased and the on current $\left(\mathrm{I}_{\text {on }}\right)$ was decreased, since the resistivity of channel and total trap density were increased. It is observed that the electrical properties of a-IGZO TFTs were different depending on the contact between the a-IGZO and S/D electrode. In the case of the device with $\mathrm{Ti} / \mathrm{Au}$ electrode, the on current was higher but the value of SS was also higher than those of a-IZO electrode. However, the a-IGZO TFT with Oxide/Metal/ Oxide (OMO) electrode showed good performance in both respects.

In order to investigate the specific contact resistance with various electrodes, the transfer line method (TLM) was used. Total resistance $\left(\mathrm{R}_{\mathrm{tot}}\right)$ was determined from the slope of the linear region of the output characteristics by using Equation (1) $[9,10]$.

$$
R_{t o t}=R_{S D}+\frac{R_{S H}}{W} L_{C H}
$$
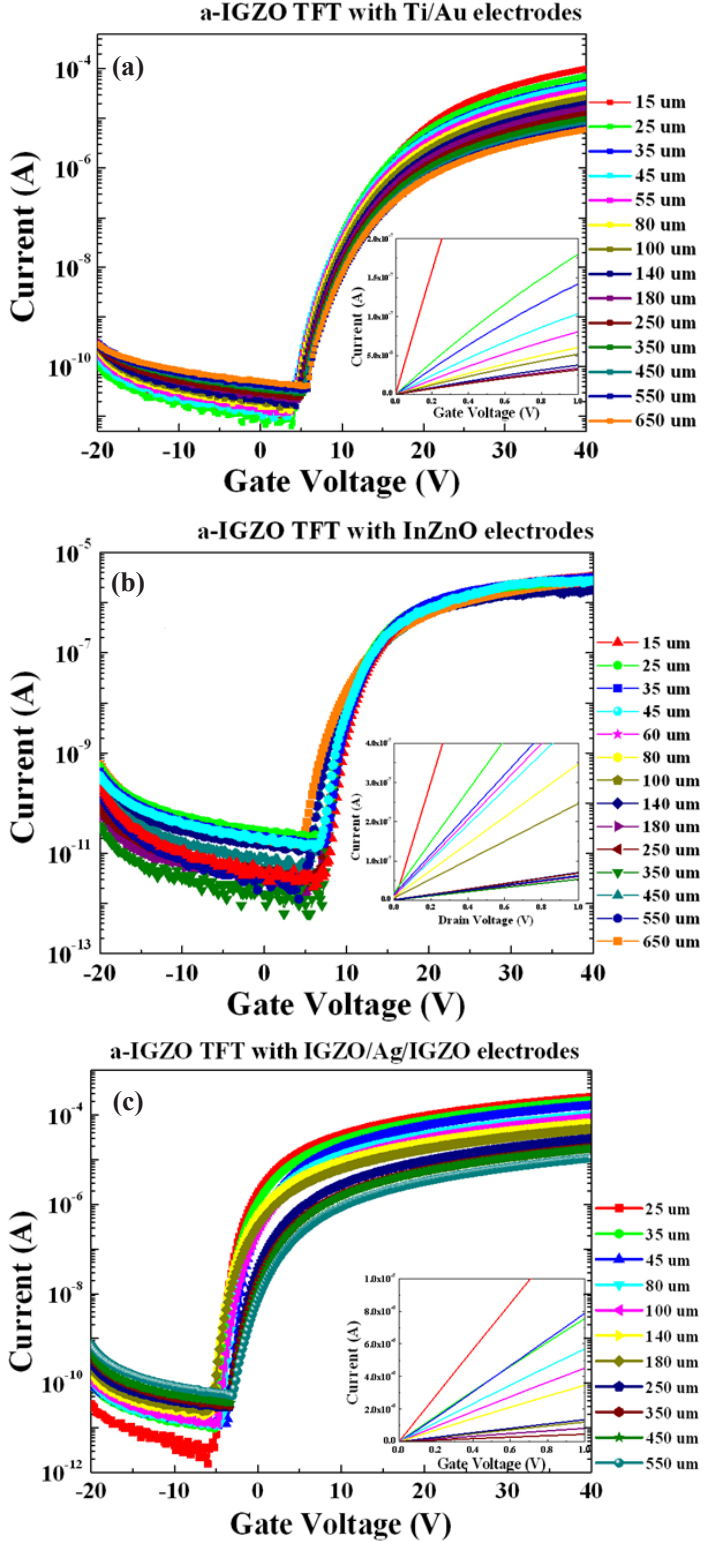

Fig. 3. Transfer curves of a-IGZO TFTs depending on $\mathrm{L}_{\mathrm{ch}}$ with (a) Ti / Au electrodes, (b) a-IZO electrodes, and (c) a-IGZO/Ag/a-IGZO electrodes at VDS $=5 \mathrm{~V}$. The inset shows output characteristics of a-IGZO TFTs measured at $\mathrm{V}_{\mathrm{GS}}=15 \mathrm{~V}$ with different $\mathrm{L}_{\mathrm{ch}} \mathrm{S}$.

where $\mathrm{L}_{\mathrm{ch}}$ is the separation of the electrodes, and $\mathrm{W}$ is the electrode width fixed at $250 \mu \mathrm{m}$, as shown in Fig. $4 . \mathrm{R}_{\text {tot }}$ is a function of the contact resistances $\left(\mathrm{R}_{\mathrm{SD}}\right)$ of the two contacts and the sheet resistance $\left(\mathrm{R}_{\mathrm{SH}}\right)$ of the semiconducting layer outside the contact, and $\mathrm{R}_{\mathrm{SD}}$ and $\mathrm{R}_{\mathrm{SH}}$ were obtained from the $\mathrm{y}$-axis intercept and slope the $\mathrm{R}_{\text {tot }}-\mathrm{L}_{\mathrm{ch}}$ plot of Fig. 5, respectively.

Figure 5 shows total resistance variation as a function of channel length depending on various electrodes. The $\mathrm{R}_{\mathrm{SD}}$ with $\mathrm{Ti} / \mathrm{Au}$ was higher than that with the a-IZO, and IGZO/Ag/IGZO electrodes and the ratio of $R_{S D} / R_{S H}$ were also higher. This is mainly due to low energy barrier between the channel and S/D electrodes, so the oxide electrodes were easy for injecting the carriers into channel layer. Therefore, the IGZO/Ag/IGZO electrodes showed low contact resistance for S/D electrodes of a-IGZO TFTs. 


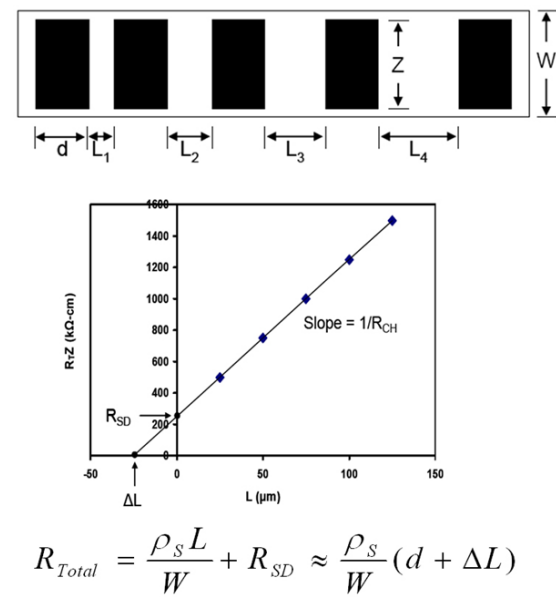

Fig. 4. Explanation and equation of transmission line method to derive total resistance and channel resistance.

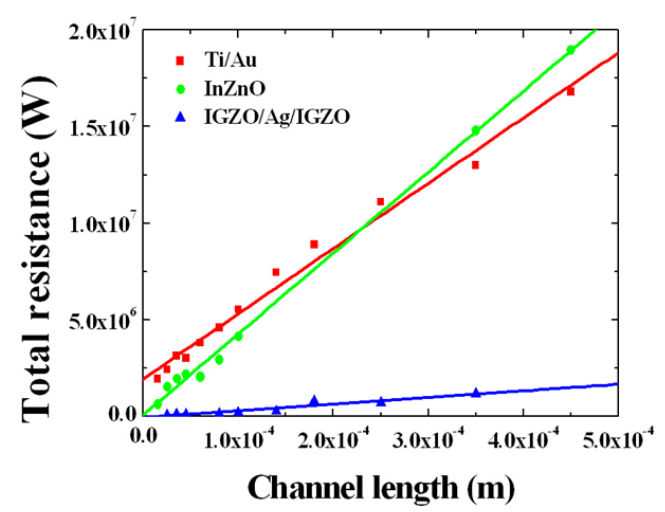

Fig. 5. Plot of $\mathrm{R}_{\text {tot }}$ Vs. $\mathrm{L}_{\text {ch }}$ for TFTs with different $\mathrm{S} / \mathrm{D}$ electrodes.

\section{CONCLUSIONS}

In conclusion, different $\mathrm{S} / \mathrm{D}$ electrodes of the a-IGZO TFTs have been compared by TLM. The electrical properties and stability of a-IGZO TFT showed a different trend with S/D electrodes. The a-IGZO TFTs with a-IGZO/Ag/a-IGZO of S/D elec- trodes had a good performance and a low contact resistance due to the homo-junction with channel layer. It is clearly observed that the $\mathrm{R}_{\mathrm{SD}}$ with $\mathrm{Ti} / \mathrm{Au}$ was higher than that with the a-IZO and IGZO/Ag/IGZO electrodes, and the ratio of $R_{S D} / R_{S H}$ was also higher. This is mainly due to low energy barrier between the channel and S/D electrodes, so the oxide electrodes were easy for injecting the carriers into channel layer. Therefore, the IGZO/ Ag/IGZO electrodes showed low contact resistance for S/D electrodes of a-IGZO TFTs.

\section{REFERENCES}

[1] K. Nomura, H. Ohta, A. Takagi, T. Kamiya, M. Hirano and H. Hosono, Nature, 432, 488 (2004). [DOI: http://dx.doi.org/10.1038/ nature03090].

[2] K. Nomura, H. Ohta, K. Ueda, T. Kamiya, M. Hirano, and H. Hosono, Science, 23, 1269 (2003). [DOI: http://dx.doi.org/10.1126/ science.1083212].

[3] J. S. Park, K. S. Kim, Y.-G. Park, Y. G. Mo, H. D. Kim, and J. K. Jeong, Adv. Mater., 21, 329 (2009). [DOI: http://dx.doi. org/10.1002/adma.200802246].

[4] E. Chong, S. H. Kim, and S. Y. Lee, Appl. Phys. Lett., 97, 252112 (2010). [DOI: http://dx.doi.org/10.1063/1.3530453].

[5] K. H. Choi, Y.Y. Choi, J. A Jeong, H.K. Kim, and S. Jeon, Electrochemical and Solid-State Letters, 14, 152 (2011). [DOI: http:// dx.doi.org/10.1149/1.3533436].

[6] P. Barquinha, A. M. Vilà, G. Gonçalves, L.Pereira, R. Martins, J. R. Morante, and E. Fortunato, IEEE Transactions on Electron Devices, 55, 4, (2008). [DOI: http://dx.doi.org/10.1109/ TED.2008.916717].

[7] K. Nomura, T. Kamiya, H. Yanagi, E. Ikenaga, K. Yang, K. Kobayashi, M. Hirano, and H. Hosono, Appl. Phys. Lett., 92, 202117 (2008). [DOI: http://dx.doi.org/10.1063/1.2927306].

[8] R.B.M. Crossa and M. M. De Souza, Appl. Phys. Lett., 89, 263513 (2006). [DOI: http://dx.doi.org/10.1063/1.2425020].

[9] K. Jeon, C. Kim, I. Song, J. Park, S. Kim, S. Kim, Y. Park, J. H. Park, S. Lee, D. M. Kim, and D. H. Kim, Appl. Phys. Lett., 93, 182102 (2008). [DOI: http://dx.doi.org/10.1063/1.3013842].

[10] S. Lee, H. Park, and D. C. Paine, Appl. Phys., 109, 063702 (2011). [DOI: http://dx.doi.org/10.1063/1.3549810].

[11] R.B.M. Cross and M. M. De. Souza, Appl. Phys. Lett., 89, 263513 (2006). [DOI: http://dx.doi.org/10.1063/1.2425020]. 